\begin{tabular}{|c|c|c|}
\hline Beitr. Ent. & Keltern & ISSN 0005-805X \\
\hline $\mathbf{5 1 ( 2 0 0 1 ) 2}$ & S. $375-391$ & 16.12 .2001 \\
\hline
\end{tabular}

\title{
Taxonomische Studien zur Hydrophiliden-Gattung Enochrus THOMSON, 1859. 2. Teil: Die afrikanischen Arten der Untergattung Methydrus REY, 1885. A: Die Enochrus meracus-Gruppe
}

\section{(Coleoptera, Hydrophilidae)}

Mit 15 Figuren

FRANZ HEBAUER

Summary

Phylogenetic reasons in conflict with typologic reasons within the genus Enochrus are caused by the multiple overlapping of the subgenera of that genus. Therefore BALFOUR-BROWNE (1958) proposed to facilitate the taxonomic work by subdividing the genus in species groups undependent from phylogenetic viewpoints. Following this way 3 species new to science: E. ituriensis sp. n., E. melinus sp. n., E. mergus sp. n. are described. A key to the African species of the E. meracus-group is given and the aedeagi of all known species are figured.

\section{Key words}

Insecta, Coleoptera, Hydrophilidae, Enocbrus, systematics, taxonomy, Africa, key, new species.

\section{Zusammenfassung}

In der Fortsetzung der taxonomischen Studien zur Hydrophiliden-Gattung Enochrus THouson wird in vorliegendem 2. Teil die Enocbrus meracus-Gruppe revidiert und um 3 neue Arten (ituriensis, melinus, mergus) aus Afrika erweitert. Eine Bestimmungstabelle der afrikanischen Arten und die Abbildungen der Aedoeagi aller bisher bekannten Arten dieser Gruppe sollen die Zuordnung erleichtern.

\section{Einleitung}

Die über alle Erdteile verbreitete Gattung Enocbrus THOMSON zählt schon allein schon aufgrund der großen Artenzahl von derzeit 175 beschriebenen Arten (n. HANSEN 1999) mit zahlreichen ungeklärten Synonymen zu den taxonomischen Problemgruppen unter den Hydrophilidae. Hinzu kommt die geringe Differenzierung sowohl in der Genitalmorphologie wie auch in Habitus und Skulptur, so daß dem Taxonomen verständlicherweise nur wenige brauchbare Bestimmungstabellen und noch weniger verläßlich determinierte Vergleichsexemplare zur Verfügung stehen. Derzeit bemüht sich S. SCHÖDL, 
Wien die paläarktischen und orientalischen Arten der Gattung zu revidieren, eine wahrliche Sisyphusarbeit! Für die nearktische Fauna existieren zusammenfassende Arbeiten von R. W. GUNDERsen (1977), G. H. HORN (1890), F. N. YOUNG (1954), D. C. Muler (1964) sowie ein unveröffentlichter Bestimmungsschlüssel von J. W. GREEN \& D. C. MILLER. Die australischen Enochrus revidierte C. H. S. WATTS (1998). Bisher wenig beachtet bzw. immer gescheut wurden die afrikanischen Arten dieser Gattung. Lediglich J. BALFOUR-BROWNE leistete in seinen Untersuchungen von 1958 und 1959 Pionierarbeit in der Weise, daß er neben der Beschreibung neuer Taxa abweichend von der geltenden systematischen Gliederung der (aus den 4 in Afrika vertretenen Untergattungen Enochrus s. str., Lumetus ZAITZEV, Metbydrus REY und Hydatotrephis MACLEAY bestehenden) Gattung eine von der Taxonomie her bestimmte Gliederung in Artengruppen vornahm. Diese Gruppierung basiert einmal auf der Genitalmorphologie (ellipsoideus-Gruppe), ein anderes Mal auf der Ausbildung der Mesosternallamelle (meracus-Gruppe), was nicht immer deckungsgleich sein muß mit phylogenetischen Gesichtspunkten, dafür aber brauchbar für den Taxonomen und durchaus erweiterbar. Die klassische Einteilung in Untergattungen bringt gerade hier immer wieder Übergangsformen und Überschneidungen zu Tage, so daß die Abgrenzungen bereits mehrmals neu definiert wurden ( $z$. B. Unterg. Metbydrus - D ORCHYMONT, 1939; Synonymisierung der Untergattung Pseudenocbrus LOMNICKI etc. Einbeziehung der Untergattung Hydatotrephis MACLEAY usw.). So erlitt die Untergattung Metbydrus bisher folgende Definitionen:

- REY, 1884: Pronotum lateral ohne Reihe größerer systematischer Punkte;

- ORCHYMONT, 1939: Analsternit mit bewimperter apikaler Ausrandung;

- HANSEN, 1990: Palpenendglied kürzer als das vorletzte Glied, Analsternit mit apikaler Austandung.

BALFOUR-BROWNE (1958) hält auch diese Kriterien nicht für konstant. Tatsächlich ist beim Vergleich mehrerer Arten eine Variabilität beginnend bei einem tief halbkreisförmigen Ausschnitt über einen flachen, bewimperten Eindruck bis zur völligen Reduktion des Ausschnitts zu beobachten. Die relative Länge des Palpenendglieds variiert gerade innerhalb der E. meracus-Gruppe am meisten.

Erneute Definition der bestehenden Untergattungen würden erfahrungsgemäß das Problem nicht lösen, nut noch mehr Verwirrung stiften, obwohl genügend Anlaß dazu gegeben ist, so wird E. latus KUwERT, derzeit als Metbydrus betrachtet, könnte aber ebenso (wegen der gleichlangen 3. und 4. Palpenglieder) zu Enochrus s.str. gestellt werden. E. mediastinus BALFOUR-BROWNE zeigt durch die rudimentär apikalen Punktreihen der Elytren einen Übergang der Untergattung Lumetus zu Holcophilydrus (in der Madagassis verbreitet) an usw. Die Ausprägung der Mesosternallamelle bei allen Arten der E. meracusGruppe erweckt ganz und gar nicht den Eindruck einer bloßen Konvergenz, vielmehr den einer Reduktion oder Synapomorphie. Selbst für den Fall, daß dies ein Trugschluß wäre, sollte man aufgrund der Uniformität innerhalb dieser Gruppe aus praktisch-taxonomischen Gründen der Typologie den Vorrang vor der Phylogenie einräumen.

Wegen der schwierigen Zuordnung der meisten Arten der E. meracus-Gruppe nach äuBeren Merkmalen ist die Genitalpräparation aller verfügbaren Männchen unerläßlich. 


\section{Danksagung und Abkürzungen}

Allen Institutionen und Kollegen, die bereit waren Material für die vorliegende Arbeit zur Verfügung zu stellen sei an dieser Stelle herzlich gedankt.

$\begin{array}{ll}\text { CHG } & \text { Coll. F. Hebauer, Grafling, } \\ \text { CMP } & \text { The Carnegie Museum Pittsburgh (R. L. DAVIDSON), } \\ \text { MHNG } & \text { Museum d'Histoire Naturelle Genève (I. LÖBL) } \\ \text { MHNP } & \text { Muséum national d'Histoire naturelle, Paris (Y. CANBEFORT) } \\ \text { MKB } & \text { Museum und Forschungsinstitut Alexander Koenig, Bonn (M. SCHMTT), } \\ \text { MNB } & \text { Museum für Naturkunde an der Humboldt-Universität, Berlin (M. UHLIG), } \\ \text { MNS } & \text { Staatl. Museum für Naturkunde Stuttgart (W. SCHAWALL.ER) } \\ \text { NHM } & \text { British Museum (Natural History), London (S. HINE) } \\ \text { NME } & \text { Naturkundemuseum Erfurt (M. HARTMANN), } \\ \text { SMWN } & \text { State Museum of Windhoek, Namibia J. ANJOS DIAS, A. H. KIRK-SPRIGGS) } \\ \text { TMB } & \text { Természettudományi Múzeum Budapest (O. MERKL, G. SZÉL) } \\ \text { ZML } & \text { Zoological Museum Lund (R. DANIELSSON) }\end{array}$

\section{Systematik}

Zur E. meracus-Gruppe zählen nach BALFOUR-BROWNE (1958), small species of under $4 \mathrm{~mm}$ in length with the mesosternal lamina low, more or less regularly arcuate and without trace of mucro", Arten nach der Definition von D ORCHYMONT (1939) für die Untergattung Methydrus, deren Mesosternallamelle als niedriger bogenförmiger Längskiel ohne jedes Zähnchen ausgebildet ist (Fig. 12).

Alle Arten dieser Gruppe zeigen die für Methydrus charakteristische winzige bewimperte apikale Ausrandung des Analsternits.

Ein gewisses Problem in diesem Zusammenhang stellt E. pellax BALFOUR-BROWNE, 1958 dar. Der Autor selbst stellt die Art aufgrund der gleichen Länge der 3. und 4. Glieder der Maxillarpalpen und des fehlenden bewimperten Ausschnitts am Hinterrand des Analsternits zu Enochrus s. str., ordnet sie aber gleichzeitig in seine zur Untergattung Metbydrus REY zählende E. meracus-Gruppe ein (wegen der bogenförmigen Mesosternallamelle ohne Zähnchen). Eine versuchte Zuordnung (HEBAUER 1998) anhand eines vermeintlich zu E. pellax gehörigen Exemplars mit angedeutetem Zähnchen am apikalen Ende der Mesosternallamelle sowie einem flach ausgerandetem Analsternit (bei ansonsten voller Übereinstimmung mit der Beschreibung von E. pellax) erwies sich inzwischen als Irrtum. Bei dem diskutiertem Exemplar handelt es sich um eine noch unbeschriebene Art, die zu einem späteren Zeitpunkt benannt werden soll. Ein Typenvergleich war bisher nicht möglich, da der Holotypus im Museum d'Histoire Naturelle Paris nicht erreichbar ist, jedoch konnte inzwischen ein unzweifelhaftes Männchen von E. pellax aus Nigeria untersucht werden. Aus taxonomischen Gründen soll E. pellax im folgenden im Bestimmungsschlüssel der E. meracus-Gruppe verbleiben, auch in der Überzeugung, daß diese Art künftig zur Untergattung Metbydrus zu stellen ist. Bei weiteren Arten der $E$. meracus-Gruppe mit deutlicher apicaler Ausrandung des Analsternits sind nämlich ebenfalls die 3. und 4. Glieder der Maxillarpalpen von annähernd gleicher Länge, meist auch relativ kurz, ein Merkmal, das sie der Untergattung Enochrus s. str. sehr nahebringt. 
Die meisten Arten der E. meracus-Gruppe sind über Westafrika verbreitet, wenige bis Ost- und Südafrika; einige weitere Arten treten in der Orientalis (HEBAUER, 1995) und Nearktis auf. Im übrigen sind auch die Arten der E. ellipsoideus-Gruppe (sensu BALFOUR-BROWNE 1959) sowohl über die Alte wie die Neue Welt verbreitet.

Eine genitalmorphologische Besonderheit innerhalb dieser Gruppe, die geeignet ist, die Bestimmung zu erleichtern zeigt sich bei E. furculus. Hier weicht das männliche Genital von allen anderen Arten der Gattung insofern ab, als der Medianlobus nur einteilig ist (ohne Ventralplatte) und die Parameren an der Innenseite gabelig aufgespalten sind.

\section{Bestimmungstabelle}

1 Analsternit am Hinterrand ohne bewimperte Ausrandung; Maxillarpalpen relativ kurz, das vorletzte und letzte Glied etwa gleich lang; völlig schwarze Art; 3,1 mm E. pellax BALFOUR-BROWNE, 1958

Analsternit am Hinterrand mit deutlicher bewimperter Ausrandung ................... 2

2 Oberseite hell-gelbbraun, kleinere Arten unter 3,3 $\mathrm{mm}$.................................... 3

Oberseite pechbraun-schwarz .............................................................................. 4

3 Letztes Glied der Maxillarpalpen deutlich kürzer als das vorletzte (14:17); Nahtintervall geschwärzt; $3,0 \mathrm{~mm}$.............................................. E. melinus sp. n.

Letztes Glied der Maxillarpalpen kaum kürzer als das vorletzte (11:12); ähnlich E. natalensis G./H.! 2.8-3.0 mm ....... E. petersenae BALFOUR-BROWNE, 1958

$4 \quad$ Labrum hell ................................................................................................... 5

Labrum schwarz, zumindest in der Mitte geschwärzt ........................................... 8

5 Pronotum mäßig kräftig und dicht punktiert; letztes Glied der Maxillarpalpen viel kürzer als das vorletzte (8:12). Aedoeagus ohne Ventralplatte! Parameren mit je einem langen Dorn auf der Innenseite; 3,2-3,5 $\mathrm{mm}$.

E. furculus BALF.-BR., 1958

Pronotum sehr fein und sehr locker punktiert; letztes und vorletztes Glied der Maxillarpalpen annähernd gleichlang

Größere Art: $3,3 \mathrm{~mm}$; letztes Glied der Maxillarpalpen nur wenig kürzer als das vorletzte (9:10); Clypeus basal dreieckig, selten ausgedehnter geschwärzt. Medianlobus des Aedoeagus breit spatelförmig, fast so lang wie die Parameren, diese apikal häkchenförmig nach außen gekrümmt.

E. merops BALFOUR-BROWNE., 1958

Kleinere Arten: 2,2-2,5 mm; letztes und vorletztes Glied der Maxillarpalpen gleichlang

7 Clypeus basal diffus dreieckig geschwärzt. Medianlobus des Aedoeagus fadenförmig. so lang wie die Parameren; diese apikal gerade auslaufend.

E. rutilus BALFOUR-BROWNE, 1958

Clypeus in der Mitte mit fast parallelem schwarzen Längsband. Medianlobus des Aedoeagus schlank keulenförmig mit annähernd gleichlanger Ventralplatte; Paramerenspitzen häkchenförmig nach außen gekrümmt.

E. mergus sp. $\mathrm{n}$. 
8 Elyten mit gut erkennbaren unregelmäßigen Reihen gröberer Punkte; 3,5-3,8 mm

Elytren ohne deutlich erkennbare gröbere Punktreihen; zumindest basal pechschwarz; 3,0-4.0 $\mathrm{mm}$

9 Elytren mit 4 deutlichen unregelmäßigen gröberen Punktreihen; die Basis mit einer gut begrenzten großen schwarzen Dreieckmakel. Labrum nur in der Mitte angedunkelt. Clypeus mit basaler dreieckiger Schwärzung. Das letzte Glied der Maxillarpalpen viel kürzer als das vorletzte (15:20); Medianlobus lang und schmal, Parameren im distalen Drittel nicht abrupt verjüngt; $3,5-3,8 \mathrm{~mm}$.

E. capucinus HEBAUER, 1988

Elytren mit 3 weniger deutlichen unregelmäßigen gröberen Punktreihen; gleichförmig braun, mit schwarzen Nahtintervallen und schwarzen Schultermakeln. Letztes Glied der Maxillarpalpen viel kürzer als das vorletzte (10:15). Labrum schwarz, Clypeus mit breitem schwarzen Medianband. Parameren im apikalen Drittel abrupt verengt; Medianlobus parallelseitig, etwa doppelt so breit wie die Parameren im distalen Drittel; 3,5 mm............ E. anticus (RÉGIMBART, 1905)

10 Durchschnittlich kleiner: 3,0-3,5 mm; Labrum schwarz; Clypeus mit breitem schwarzen, fast parallelseitigem Medianband; Elytren basal mit großer, unscharf begrenzter Dreieckmakel und dunklem Schulterwisch. Letztes Glied der Maxillarpalpen nur wenig kürzer als das vorletzte (14:16). Medianlobus des Aedoeagus schmal parallelseitig, apikal leicht zugespitzt, deutlich kürzer als die Parameren; Ventralplatte etwa halb so lang wie der Lobus.

E. meracus BALFOUR-BROWNE, 1952

Durchschnittlich größer: 3,5-4,0 mm; Labrum nur in der Mitte geschwärzt, Clypeus mit breit trapezförmigem schwarzen Medianband; Elytren bis kurz vor dem aufgehellten Apex pechschwarz. Letztes Glied der Maxillarpalpen viel kürzer als das vorletzte (10:15). Medianlobus des Aedoeagus fast fadenförmig dünn, so lang wie die Parameren, scharf zugespitzt; Ventralplatte nur 1/3 der Länge des Lobus. E. ituriensis sp. n.

\section{Die Arten}

\section{Enochrus (Methydrus) anticus (RÉGIMBART, 1905)}

1905 Pbilbydrus anticus REGIMBART, p. 214.

Locus typicus: Äthiopien (Asmara).

Typenmaterial: Holotypus (MHNP).

\section{Nachweise:}

Äthiopien: Ethiopia, Addis Abbeba, 16.9.1980, leg. Demeter (CHG).- Ethiopia, Bahar Dar. 7.10., 8.10., 15.10., 19.10., 20.10., 22.10., 29.10., 31.10.1968 K. W. u. H. Harde leg., Lichtfang. (MNS, CHG); ibidem: 15.-25.10.1967, leg. H. Schütz (MNS, CHG); ibidem: VII.1968, leg. O. Sebald (MNS).- Ethiopia: Lake Langano, at light, 15.10.1980, Nr. 243, leg. A. Demeter (MNS).- Ethiopia: Arsi Assella, 1.-31-1-1989, $2400 \mathrm{~m}$, ibidem 4.2.1989, 2600 m, leg. S. Persson (ZML).- Gabon: Gabon, Makokou, 4.1971, J. Mateu (MHNG).Ghana: Ghana, Kumasi, 6.7.1967; 1 Ex.: 18.5 .1967 leg. Endrödy.-Younga (MHNG).- Kenia: Kenya: Nairobi, at light, in Garden with stream at Hotel Boulevard 9.-12.12.1978, leg. L. Cederholm (ZML).- Mada- 
gaskar: Madagaskar East, $5 \mathrm{~km} \mathrm{~S}$ Ampamoho pr. Andilamena, 950-1000 m, 28.12.-20.1.1995, lux, G. Dunay \& J. Janak, (MNB).- Republik Südafrika: Natal, Cathedral Peak, Mikes Pass, $1700 \mathrm{~m}, 24.2 .1984$, Moolman (Mus. Pretoria); Natal: Oribi Gorge N. R. Port Shepstone Area, 30³7'S-3009'E, 28.12.1988, leg. E. Holm \& E. Marais, belt trap (SMWN); Transvaal, Rusterberg Nat. Res., 17.-20.3.1980, HarropMoolman (Mus. Pretoria); Tvl. Rusterberg Nature Res. $25^{\circ} 40^{\prime} \mathrm{S}-27^{\circ} 12^{\prime} \mathrm{E}, 12 .-20.3 .1980$, leg. W. A. Harrop \& C. G. E. Moolman (Mus. Pretoria).- Swaziland: Mliwwane wildlife Sanctuary $26^{\circ} 29^{\prime} 22^{\prime \prime} \mathrm{S} 31^{\circ} 11^{\prime} \mathrm{E}, 800 \mathrm{~m}$, 17.-19.11.1996, leg. M. Hartmann (NME); Basutoland, nr. Quthing, 16.3.1951, leg. Brinck-Rudebeck (ZML).Uganda: Uganda, Distr. Masindi. Budongo Forest n. Sonso, $1^{\circ} 45^{`} \mathrm{~N}-31^{\circ} 35^{\top} \mathrm{W}, 19 .-30.6 .1995$, leg. Th. Wagner (MKB).- Sambia: Kafue River NP, Chunga Camp, lux, $15^{\circ} 02^{\prime} 35^{\prime \prime S}-26^{\circ} 00^{\prime} 09^{\prime \prime} \mathrm{E}$, 29.3.1993, leg. M. Uhlig (MNB); Zambia: $15^{\circ} 02^{\prime} 35^{\prime \prime S} 26^{\circ} 00^{\prime} 090^{\circ} \mathrm{E}$, Kafue NP Chunga Camp, lux, 27.3.1993, leg. M. Uhlig (MNB); Zambia, $5 \mathrm{~km} \mathrm{~S}$ Choma, 16⒋ $49^{\prime} 14^{\prime \prime S} \mathrm{~S}-26^{\circ} 55^{\prime} 44^{\prime \prime} \mathrm{E}, 15.3 .1993$, lux, leg. M. Uhlig (MNB).

Wiederbeschreibung: 2,8 × 1,9 mm.- Länglich oval, glänzend, stark gewölbt, Oberseite rötlich braun.

Kopf schwarz mit zwei ziemlich großen hellen Präokularmakeln, mäßig dicht punktiert; Labrum schwarz, Maxillarpalpen einfarbig gelb (Fig. 13d), Fühler gelb mit angedunkelter Endkeule. Pronotum auf der Mitte ausgedehnt unscharf angedunkelt, wie der Kopf und die Elytren punktiert. Elytren rötlich braun mit schwarzem Nahtintervall und aufgehellten Seiten; drei unregelmäßige Reihen gröberer Punkte wenig deutlich. Beine und Tarsen rötlich braun bis gelb; Femora und Unterseite schwarz, Apikale Ausrandung des Analsternits tief, halbkreisförmig. Mesosternallamelle als sehr niedriger Bogen ohne Tuberkel ausgebildet. Aedoeagus: Parameren in der distalen Hälfte abrupt stark verjüngt, Spitzen nach innen gekrümmt. Medianlobus parallelseitig bandförmig, am Ende verrundet, fast so lang wie die Parameren (Fig. 5).

Verbreitung: O-, SO-Afrika.

\section{Enochrus (Methydrus) capucinus HEBAUER, 1988} 1988 Enocbrus (Metbydrus) capucinus HEBAUER, p. 158-159.

Locus typicus: Namibia (Nyangana/Okavango).

Typenmaterial: Holotypus ( $\left.{ }^{\star}\right)$ : Namibia, Nyangana/Okavango, 14.-22.1.1985, leg. H. Roer. (MKB).

Paratypen: 12 Ex. (dieselben Daten; MKB, CHG).

Nachweise:

Namibia: Kavango, Popa Falls, $18^{\circ} 07^{\prime} \mathrm{S} 21^{\circ} 35^{\circ} \mathrm{E}, 26.2 .-3.3 .1992$, leg. M. Uhlig (MNB).- Namibia: Kavango Mahango, Game Res. $21^{\circ} 45^{\prime} \mathrm{E} 18^{\circ} 13^{\prime} \mathrm{S}$, Picknick Site, lux, 24.11.1993, leg. M. Uhlig (MNB); Windhoek Distr. Niedersachen dam under dead $\log$ SE 2315 Bb., 4.4.1986, B. A. Curtis SM/1686 (SMWN).- Simbabwe: 1 i : Victoria Falls, Zambesi NP-Camp, $17^{\circ} 53^{\circ} \mathrm{S}-25^{\circ} 49^{\prime} \mathrm{E}, 11,-12.12 .1993$, lux, leg. M. Uhlig (MNB).

(Aedoeagus Fig. 8; Maxillarpalpus Fig. 13a).

Verbreitung: Südafrika.

Enochrus (Methydrus) furculus BALFOUR-BROWNE, 1958 1958 Enocbrus (Metbydrus) furculus J. BALFOUR-BROWNE, p. 52-53.

Locus typicus: W-Afrika (Nigeria; Ibadan).

Typenmaterial: Holotypus (đ), PT: 4 o , ibidem (NHM). 

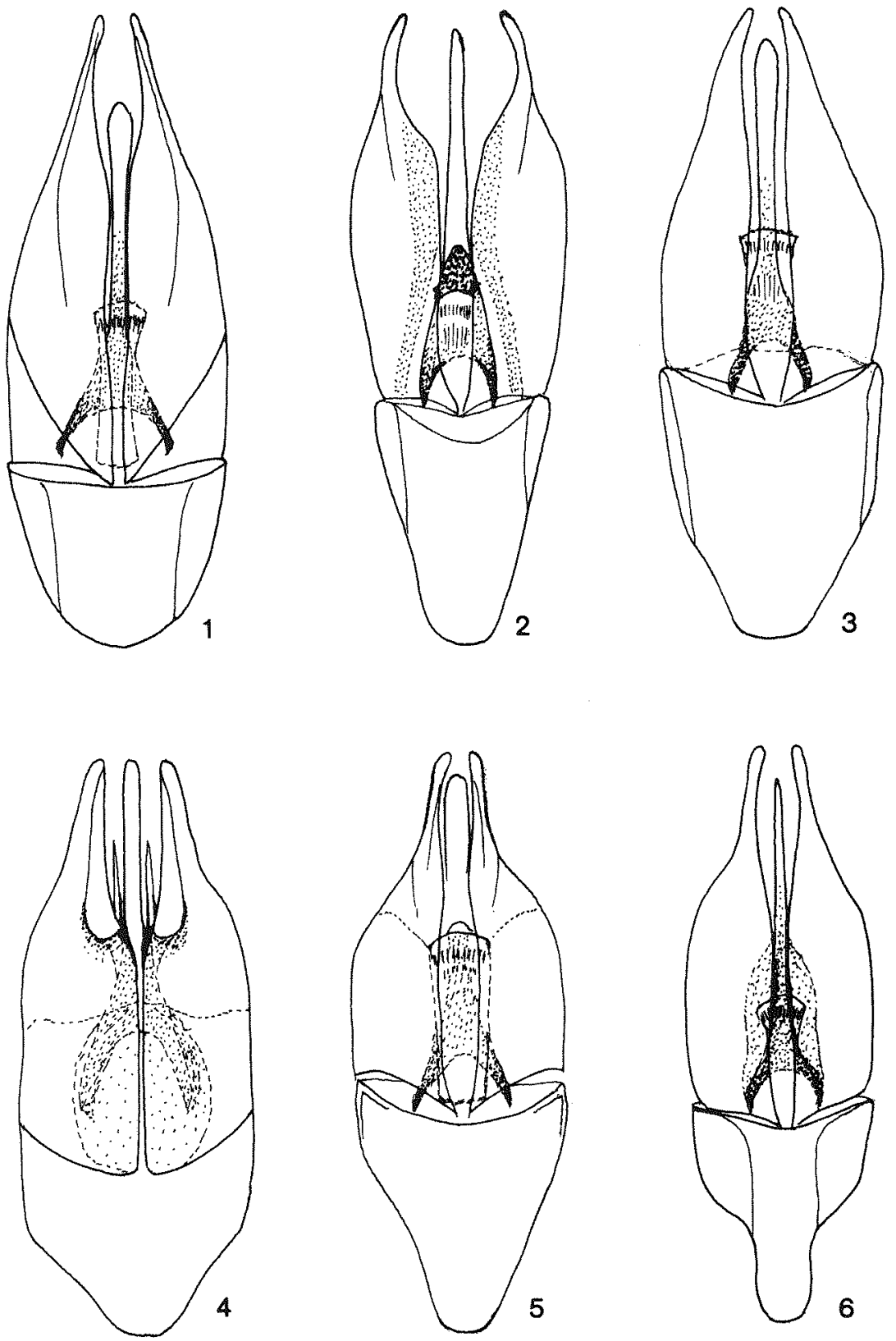

Fig. 1-6: Enochrus (Metbydrus) spp. Aedoeagus.- 1 E. pellax BALFOUR-BROWNE $(0,57 \mathrm{~mm}) ; 2$ E. melinus sp. n. $(0,57 \mathrm{~mm}) ; 3$ E. petersenae BALFOUR-BROWNE $(0,57 \mathrm{~mm}) ; 4$ E. furculus BALFOUR-BROWNE $(0,80 \mathrm{~mm}) ; \mathbf{5}$ E. anticus (RÉGIMBART), $(0,80 \mathrm{~mm}) ; 6$ E. rutilus BALFOUR-BROWNE $(0,71 \mathrm{~mm})$. 

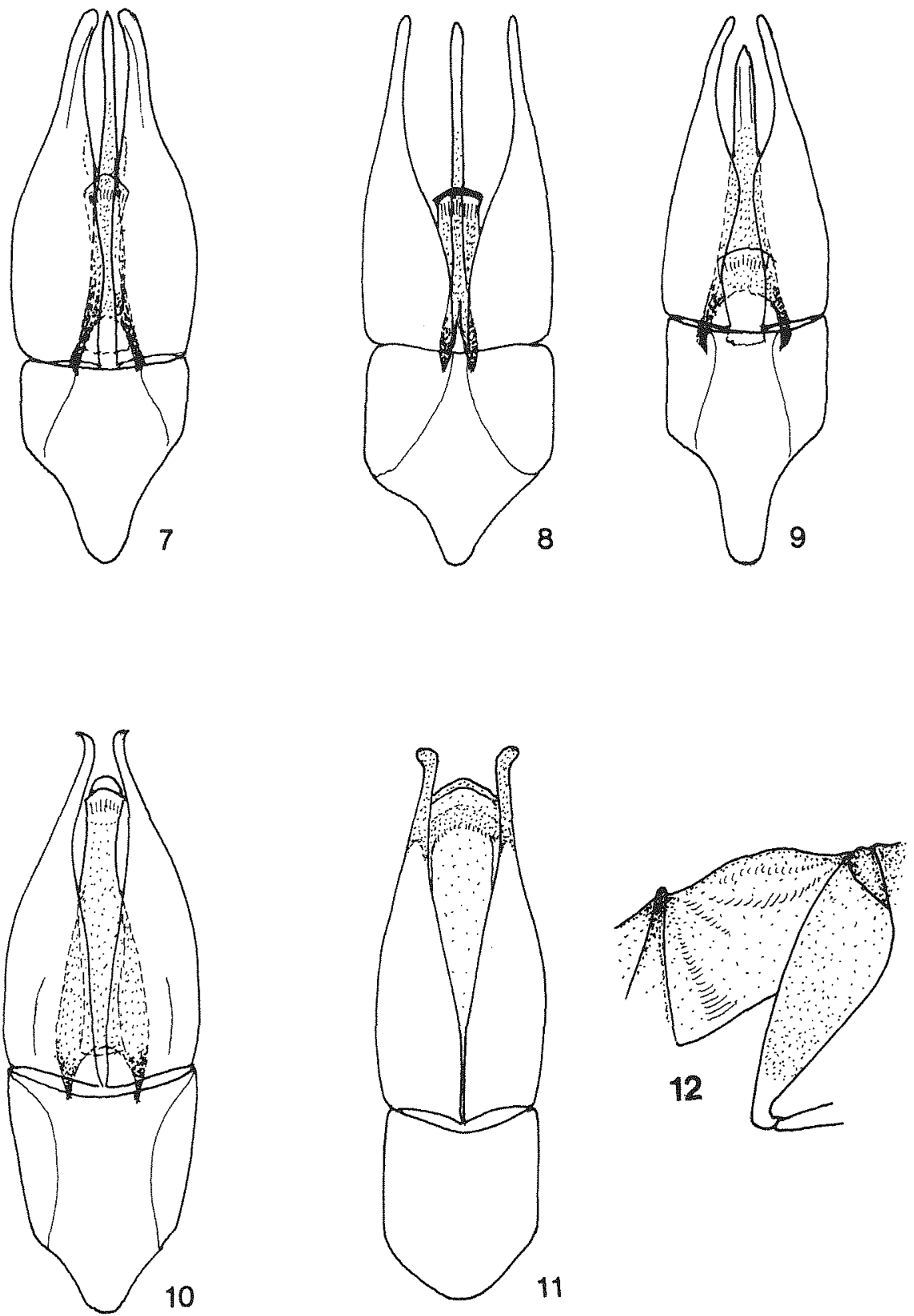

Fig. 7-11: Enochrus (Metbydrus) spp. Aedoeagus.- 7 E. ituriensis sp. n. $(0,71 \mathrm{~mm}) ; 8$ E. capucinus HebaUeR $(0,71 \mathrm{~mm}) ; 9$ Enochrus meracus BALFOUR-BROWNe $(0,71 \mathrm{~mm}) ; 10$ E. mergus sp. n. $(0,50 \mathrm{~mm}) ; 11$ E. merops BALFOUR-BROWNE (nach BALFOUR-BROWNE 1958, nachgezeichnet).

Fig. 12: Enochrus meracus BALFOUR-BROWNE, Mesosternallamelle. 

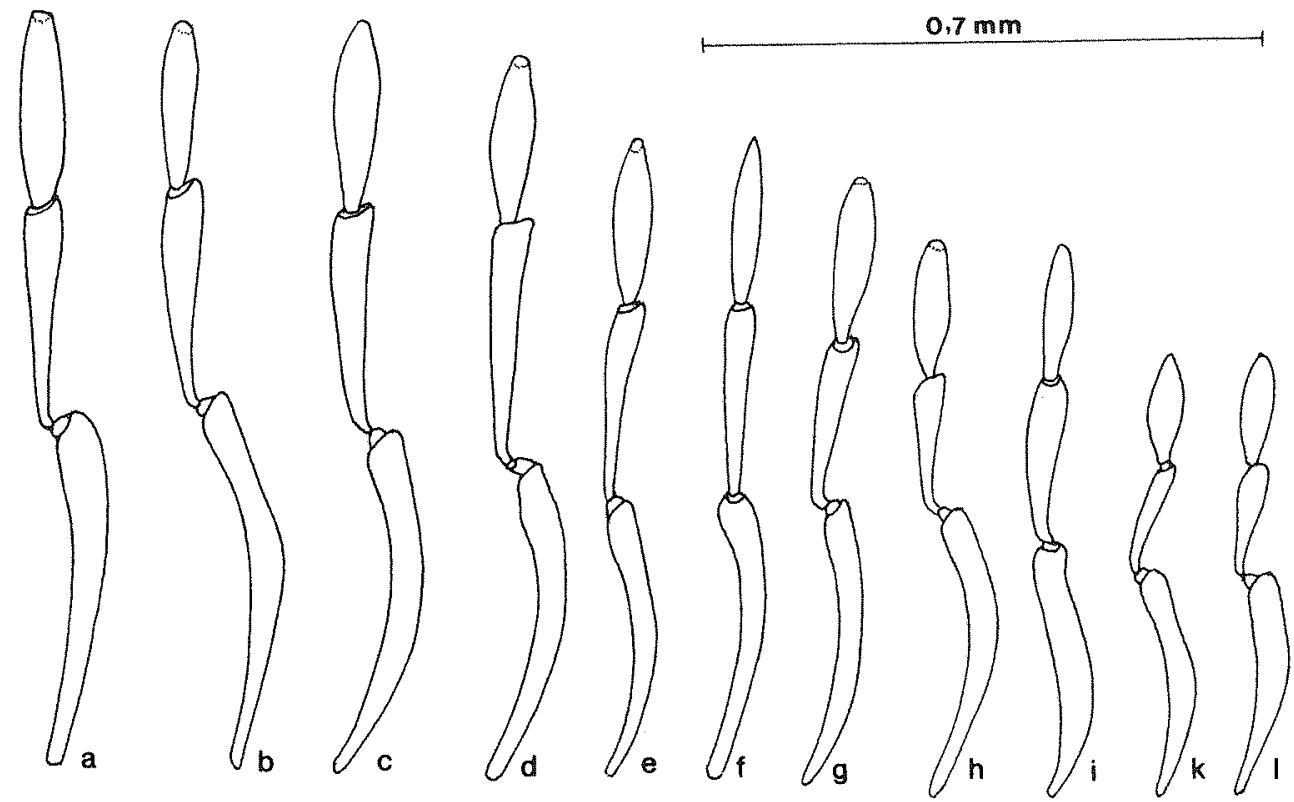

13

Fig. 13: Enocbrus spp., Maxillarpalpus, links: a) E. capuinus, b) E. furcuhus, c) E. ituriensis, d) E. anticus, e) E. melinus, f) E. merops, g) E. petersence, h) E. pellax, i) E. meracus, k) E. mergus, 1) E. rutiluss.

\section{Nachweise:}

Gabon: Makokou (à lumiére), 4./5.1971, leg. J. Mateu (MHNG); Lagune Iguélo, Ntchongarovié (forét galerie), I. 1997, leg. A. Bilardo (CHG); Lagune Iguélo, Yombé, I. 1997, leg. A. Bilardo (CHG); Lagune Iguélo, forét d’Est Tossi, I. 1997, leg. A. Bilardo (CHG); Iguélo forét, E Tossi, I.1997, leg. A. Bilardo (CHG); Rembo Bongo, M Bari Morsi, 20.6.1997, leg. A. Bilardo (CHG).- Ghana: 2 Ex.: Ashanti region, Kumasi, Nhiasu $330 \mathrm{~m}, 6^{\circ} 43^{\prime} \mathrm{N}-1^{\circ} 36^{\prime} \mathrm{W}, 20 . \mathrm{V} .1967$, at light, Nt. 218, leg. S. Endrödy-Younga (TMB); 1 Ex.: Western reg., Busua, $15 \mathrm{~m}, 4^{\circ} 48^{\prime} \mathrm{N}-1^{\circ} 56 \mathrm{~W}, 27 . \mathrm{III} .1969$, black light, Nr. 330, leg. Dr. S. Endrödy-Younga (TMB); 1 Ex.: Northern teg. Tamale, 18.VII.1970, Lichtfalle, Nr. 27, leg. Dr. S. Endrödy-Younga (TMB); 4 Ex.: Ashanti reg. Kwadaso, 320 m, $6^{\circ} 42^{\prime}$ N-1 ${ }^{\circ} 39^{\prime}$ W, 26.II.1969, black light, Nr. 314, leg. Dr. S. EndrlödyYounga (TMB); 1 Ex.: Ashanti reg., Bobiri forest tes. $320 \mathrm{~m}, 6^{\circ} 40^{\prime} \mathrm{N}-1^{\circ} 15^{\circ} \mathrm{W}, 20.111 .1966, \mathrm{Nr}$. 138, water netting, leg. Dr. S. Endrödy-Younga (TMB).- Guinea: Seredou, lux, TV.1975, leg. A. Zott (MNB; CHG).Kamerun: $5 \mathrm{~km} \mathrm{~N}$ Apouh, at light, 31.12.1977, loc. 3 (CHG); 1 o: Cameroon, $20 \mathrm{~km} \mathrm{~S} \mathrm{Edea,} 5 \mathrm{~km} \mathrm{~N}$ Apouh, at light, 31.XII.1977, Loc. 3, Lund Univ. Syst. Dept., Sweden Cameroon Exp. Dec.-Jan. 1977-78, leg. Gärdenfors-Hall-Samuelsson (ZML).- Kongo-Brazzaville: 1 : : Soil-Zoological Exp. Congo-Brazzaville, Bouenza catarract, 30.11.1963, No. 308, sifted in float, leg. Endrödy-Younga (TMB).- Nigeria: Pandam, River Li, $1300 \mathrm{~m}, 22.2 .1977$, leg. P. Beron (MHNG).- ?Sambia: 1 \%: Zambia: South Luangwa NP, Mfuwe Crocodile Farm, $13^{\circ} 06^{\circ} 03^{\prime \prime S}-31^{\circ} 47^{\prime} 32^{\prime \prime E}$, $450 \mathrm{~m}$, lux, leg. M. Uhlig (MNB).

(Aedoeagus Fig. 4, Maxillarpalpus Fig. 13b).

Verbreitung: Westafrika. 


\section{Enochrus (Methydrus) ituriensis sp. n.}

Locus typicus: Zaire (Haut Ituri).

Typenmaterial: Holotypus (đ) : 3,9 × 2,0 mm. Zaire (Haut Ituri) Nioka Mai/Sept. 1976, leg. F. Schäufele. (MNS).

Paratypen: 4 \%: (dieselben Daten; MNS, CHG).- Gabon: 10 , 9 \%: Makokou F. Ivindo rapides $32 \mathrm{~km} \mathrm{S-W}$ Loua-Loua, 3.8.1998, leg. A. Bilardo (CHG).- Liberia: 1 $\%$ : Prov. Nimba Saclepea, 26.-27.3.1988, leg. F.-T. Krell (MNS); 1 우 Bong Town, 19.3.1988, leg. F.-T. Krell (CHG).- Nigeria: 1 o: Ile-Ife, 11.7.19988, leg. F.-T. Krell (MNS).- Uganda: 3 o', 2 \%: Distr. Masindi Budongo Forest n. Sonso, $1^{\circ} 45^{`} \mathrm{~N}-31^{\circ} 35^{\prime} \mathrm{W}, 19 .-30$. VI.1995, leg. Th. Wagner (CHG).

2 Weibchen, die nicht mit Sicherheit zuzuordnen sind, werden nicht in die Typenserie einbezogen: Span. Guinea: Nkolentangan XI.1907-V.1908, G. Teßmann S. G. (CHG).

Diagnose: Kleinere Exemplare der Art sind leicht mit E. furculus und E. meracus, größere Exemplare mit E. capucinus und E. anticus zu verwechseln. Bei unausgefärbten Weibchen ist die Zuordnung kaum möglich. Nur die Kombination aller Merkmale oder das männliche Genital gewährleisten eine sichere Bestimmung.

Beschreibung: Länge: 3,5-4,0 mm.- Pechschwarz, mit seitlich breit und vorne schmal aufgehelltem Pronotum, mit großen hellen Präokularmakeln und stark verkürztem Endglied der Maxillarpalpen.

Kopf mäßig kräftig und sehr dicht punktiert, glänzend schwarz mit großen hellen Präokularmakeln; die Schwärzung des Clypeus zeigt sich in der Regel trapezförmig und erstreckt sich auf die Mitte des Labrum. Maxillarpalpen schlank, hell, das Endglied ist um ein Viertel kürzer als das vorletzte Glied (Fig. 13c). Pronotum wie der Kopf punktiert, pechschwarz, lateral breit und gut begrenzt, Vorderrand schmal gelb gesäumt. Elytren wie Kopf und Pronotum punktiert, ohne deutlich erkennbare gröbere Punktreihen, pechschwarz, nur lateral und apikal aufgehellt. Unterseite und Beine pechschwarz. Apikale Ausrandung des Analsternits halbkreisförmig. Aedoeagus mit sehr schmalem, apikal scharf zugespitzem Medianlobus von der Länge der Parameren; diese am Außenrand gleichmäßig schwach gerundet, am Innenrand lang gestreckt konkav. Ventralplatte nur ein Drittel der Länge des Medianlobus erreichend. (Fig. 7).

Derivatio nominis: Nach dem Typenfundort Haut Ituri benannt.

Verbreitung: West-, Zentralafrika.

Enochrus (Methydrus) melinus sp. n.

Locus typicus: W-Afrika (Gambia).

Typenmaterial: Holotypus ( $\left.0^{\star}\right): 3,0$ x 1,6 mm. WAfr., Gambia, Bathurst, I.1968, leg. Palm (ZML).

Paratypen: 10 Ex. (dieselben Daten; ZML).- Ghana: 1 o: North Reg: Nyankpala $15 \mathrm{~km}$ 
W Tamale, light, 1.-30.4.1970, leg. S. Endrödy-Younga (TMB, CHG); 2 q : North Reg.: Banda-Nkwanta, 26.-31.12.1965, leg. S. Endrödy-Younga (TMB, CHG); 6 o , 7 q : West Africa, Ghana, North. Reg. Nyankpala, $15 \mathrm{~km}$ W von Tamale, leg. Dr. S. Endrödy-Younga/ No. 7, Lichtfalle, 1.-30.IV.1970, (TMB).- Nigeria: 2 o, 2 o: Samaru, 20.10.1969, No. 403, light trap, leg. Dr. S. Endrödy-Younga (TMB).- Sudan: 1 Ex.: Upper Nile Prov., Malakal nr. junction Nile-Sobat, loc. D, 18.-21.9.1957, leg. Göte Forsberg (ZML); 1 o : Sudan, Disa, leg. Allison (TMB).- Tansania: 1 Ex.: Morogoro, light trap, II. 1970, leg. T. Pócs (CHG); 2 ㅇ: Tanzania: Morogoro, light trap, 20.V.1970, T. Pócs, (TMB).

Diagnose: Unter den kleinen hellen Arten der meracus-Gruppe leicht mit E. petersenae zu verwechseln; von diesem aber, außer im verschiedenen Aedoeagus, vor allem durch das stärker verkürzte Endglied der Maxillarpalpen zu unterscheiden. Von dem ähnlich aussehenden E. natalensis sind beide Arten durch die Form der Mesosternallamelle sicher zu trennen.

Beschreibung: Länge: 3.0-3,5 mm.- Von honiggelber Färbung mit basal dreieckig angedunkeltem Clypeus, bei schwarzer Stirn. Elytren mit geschwärzten Nahtintervallen und dunklen Schulterbeulen. Ganze Oberseite fein und dicht punktiert.

Kopf fein und dicht punktiert, glänzend, mit schwarzer Stirn und basal dreieckig angedunkeltem Clypeus. Labrum meist nur in der Mitte angedunkelt. Maxillarpalpen schlank; das Endglied deutlich kürzer als das vorletzte Glied (3./4. Glied = 17:14; Fig. 13e). Pronotum wie der Kopf punktiert, hell gelbbraun mit diffuser dunkler Diskalmakel; Vorderrand breit hell, Hinterrand nur um die Ecken aufgehellt. Elytren wie der Kopf punktiert; die unregelmäßigen Reihen gröberer Punkte schwer crkennbar; hell gelbbraun, glänzend, mit jeweils geschwärztem Nahtintervall und dunkler Schulterbeule. Beine und Unterseite wie die Obetseite gefärbt. Apikale Ausrandung des Analsternits halbkreisförmig. Aedoeagus mit sehr schmalem Medianlobus von der Länge der Parameren; diese basal sehr breit, erst im distalen Viertel auf der Innenseite stark konvex verjüngt. (Fig. 2).

Derivatio nominis: Lat.: melinus = honiggelb.

Verbreitung: W-Afrika.

Enochrus (Methydrus) meracus BALFOUR-BROWNE, 1952

1952 Enochrus (Metbydras) meracus J. BALFOUR-BROWNE, p. 133.

Locus typicus: W-Afrika („Dahomey“ = Burkina Faso).

Typenmaterial: Holotypus ( $\left.\sigma^{\star}\right)$, PT: 3 o, 7 q (ibidem); 1 o, 2 ㅇ: Bassila; $7 \sigma^{\star}, 6$ 우 Togo, Tohoun (NHM).

Nachweise:

Benin: Bétérou W Parakou, loc. 25, 21.7.1989, leg. B. J. v. Vondel (CHG); Ouroumonsi Nikki, loc. 11, 12.7.1989, leg. B. J. v. Vondel (CHG); Sangon W Parakou, loc. 22, 21.7.1989, leg. B. J. v. Vondel (CHG); Torou Parakou, loc. 19, 19.7.1989, leg. B. J. v. Vondel (CHG).- Botswana: Chobe Distr. Saruta Drift Camp site, $18^{\circ} 34^{\prime} \mathrm{S} 24^{\circ} 04^{\prime} \mathrm{E}, 29.12 .1988$, leg. R. D. Ward (CHG).- Burkina Faso (Holotypus).- Gambia: M Bollet, 29.1.1968, leg. T.-E. Leiler (CHG).- Ghana: Kumasi, 12.5.1967 \& 18.5.1967, leg. S. Endrödy-Younga (MHNG); Ashanti Kumasi Nhiasu, 330 m, 12.6.1967 \& 18.10.1967 \& 20.5.1967, leg. S. Endrödy-Youn- 


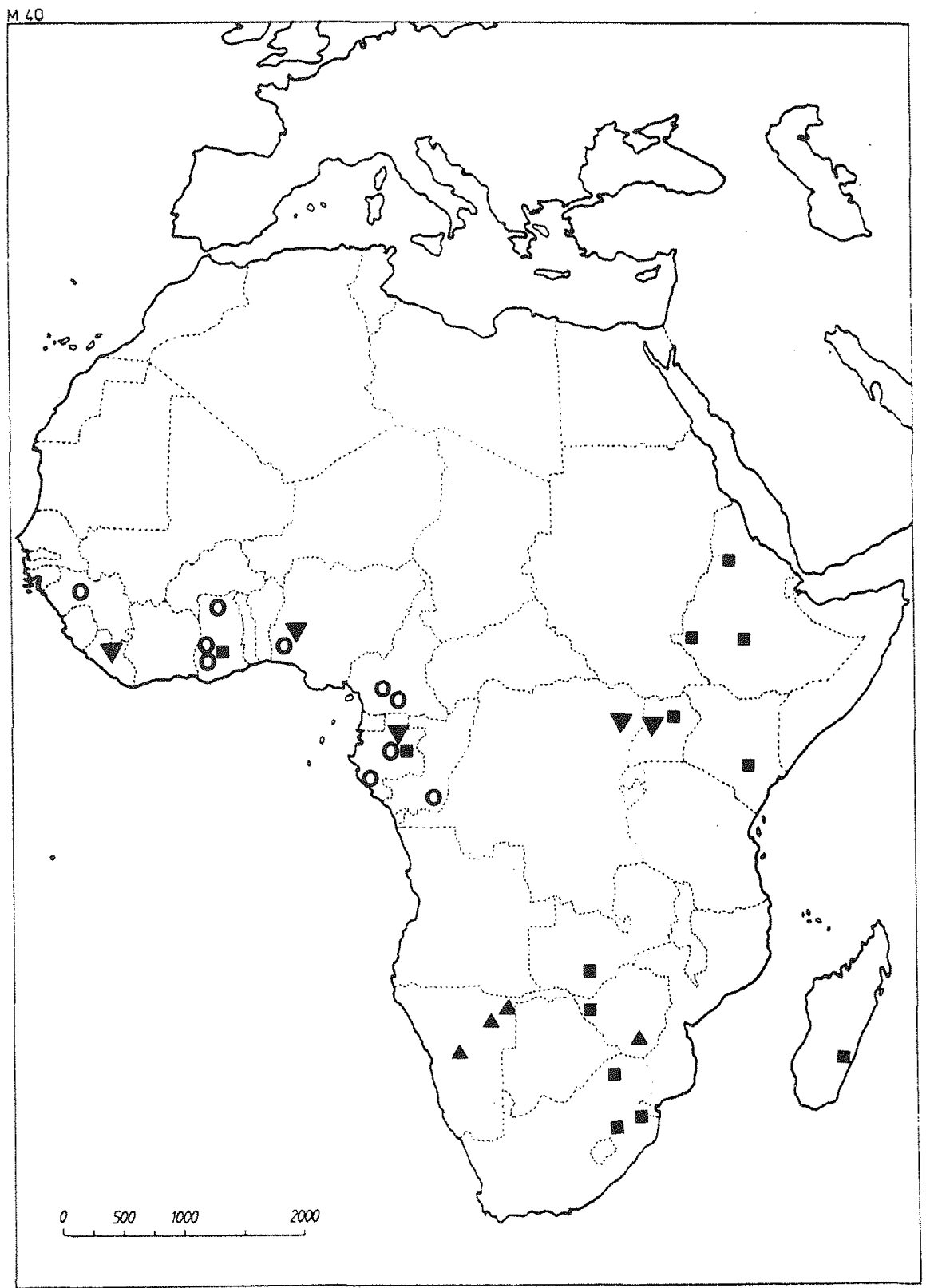

Fig. 14: Geographische Verbreitung von: E.anticus (W. Eapucinus (4), E. furculus (o), E. ituriensis (W). 
ga (MHNG, TMB); Eastern region: Boti Falls, $30 \mathrm{~km}$ NE Koforidua, shore washing, 27.12.1976, leg. S. Endrödy-Younga (MHNG, CHG); Northern Region, Banda-Nkwanta, $150 \mathrm{~m}, 8^{\circ} 22^{\prime} \mathrm{N}-2^{\circ} 08^{\prime} \mathrm{W}, 27 .-$ 29.IX.1965, No. 83, light trap, leg. Dr. S. Endtödy-Younga (TMB); Ashanti Region, Kwadaso, 330 m, $6^{\circ} 42$ N-1 39 W, No. 314, black light, 26.11.1969, leg. Dr. S. Endrödy-Younga (TMB); Ashanti Region, Bobiti forest res. $320 \mathrm{~m}, 6^{\circ} 40^{\prime} \mathrm{N}-1^{\circ} 15 \mathrm{~W}, \mathrm{No} .138,15 . \mathrm{V} .1997$, water washing, leg. Dr. S. Endrödy-Younga (TMB); Northern Region, Desongo, Mole game res., $720 \mathrm{~m}, 9^{\circ} 04 \mathrm{~N}-1^{\circ} 48^{\circ} \mathrm{W}, 12 . \mathrm{XI} .1970, \mathrm{No} .442$, light trap, leg. Dr. S. Endrödy-Younga (TMB); North. Reg., Nyankpala, $15 \mathrm{~km} \mathrm{~W}$ von Tamale, No. 7, Lichtfalle, 1.-30.IV.1970, leg. Dr. S. Endrödy-Younga (TMB); Brong Ahafo reg. Bui Camp, $130 \mathrm{~m}, 8^{\circ} 17^{\prime} \mathrm{N}-2^{\circ} 15^{\prime} \mathrm{W}$, No. 101, light trap, 1.- 4.XII.1965, leg. Dr. S. Endrödy-Younga (TMB); Northern reg. Boti falls, $30 \mathrm{~km} \mathrm{NE}$ Koforidua, No. 490, 27.XII.1971, shore washing, leg. Dr. S. Endrödy-Younga (TMB).- Guinea: Seredou, 4.5.1975, leg. A. Zott (MNB).- Nigeria: Ogoja County Rev. Meyer A9/86, stagnat pool, April 1962 (CHG); $10^{*}$ : Ile-Ife, 13.7.1988, leg. F.-T. Krell (CHG, MNS).- Sambia: $15^{\circ} 49^{\prime} 03^{\prime \prime}$ S $28^{\circ} 12^{\prime} 05^{\text {c }} \mathrm{E}, 18.3 .1993$, leg. M.

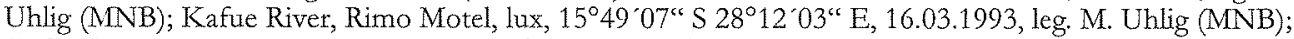

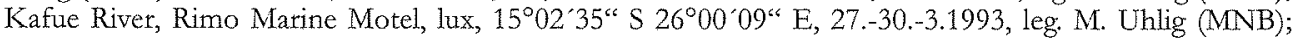
Kafue NP, Chunga Camp, Kafue River, leg. Uhlig (MNB).- Sierra Leone: Makeni $12^{\circ} 03^{\prime} \mathrm{W} 08^{\circ} 53^{\prime} \mathrm{N}$, 28.11.1993, loc. 9, light trap, 18-21; Lund University Sietra Leone Exp. 1993, leg. Cederholm-DanielssonHall (ZML).- Rep. Südafrika: O. F. S. Deelfontein 482, Bothaville SE 2736 Ba, 13.-17.11.1978, leg. A. Strydom (CHG).- Tansania: Morogoro, light trap, 20.V.1970, leg. T. Pócs (TMB).- Togo: (Paratypen).?Uganda: $10^{\circ}$ (ganz hell): Distr. Masindi, Budongo Forest n. Souso, $1^{\circ} 45^{\prime} \mathrm{N} 31^{\circ} 35^{\prime} \mathrm{W}, 19 .-30.6 .1995$, leg. Th. Wagner (MKB).

(Aedoeagus Fig. 9; Maxillarpalpus Fig. 13i).

Verbreitung: W-, SO-, S-A frika.

\section{Enochrus (Methydrus) mergus sp. n.}

\section{Locus typicus: W-Afrika (Kamerun).}

Typenmaterial: Holotypus ( $\left.8^{7}\right): 2,5 \times 1,4 \mathrm{~mm}$. Cameroon, $65 \mathrm{~km}$ NE Kribi, Bidjouka, Secondary Forest at stream (at light), 4.1.1978, Loc. no. 13., Lund University Syst. Dept./ Sweden Cameroon Exp. Dec.-Jan. 1977-78, Gärdenfors-Hall-Samuelsson. (ZML).

Paratypen: 15 Ex. (dieselben Daten; ZML).- Elfenbeinküste: Parc Nat. du Banco N Abidjan, 7.4.1988. (MNS).- 1 o : Adiopodoume, 5.5.1988, leg. F.-T. Krell (MNS).- Gabon: 17 Ex.: Makokou, lux, IV.1971, leg. J. Mateu (MHNG, CHG).- Ghana: Ashanti reg., Kumasi Nhiasu 330 m, $6^{\circ} 43^{\prime}$ N-1 ${ }^{\circ} 36^{\prime}$ W, 20.V.1967, at light, Nr. 218, leg. Dr. S. EndrödyYounga (T'MB). - Kamerun: $1 \mathrm{~s}^{\text {* }}: 40 \mathrm{~km}$ SW Messamena, Somalomo, Secondary Forest and Plantation, at light, 9.-10.1.1978, Loc. 21/ Lund University Systematic Department, Sweden Cameroon Exp. Dec.- Jan. 1977-78, leg. Gärdenfors-Samuelsson-Hall. (ZML).Kongo-Brazzaville: 5 Ex.: Soil-Zool. Exp., Bouenza catarract, 30.11.1963, No. 308, sifted in float, leg. S. Endrödy-Younga (MHNG, CHG, TMB).- Uganda: 1 q: Semliki Forest, $0^{\circ} 48^{`} \mathrm{~N} 30^{\circ} 08^{`} \mathrm{E}, 5 .-12.2 .1997$, leg. Th. Wagner (CHG).

Diagnose: Unter den kleinen (2,5 mm) schwarzen Arten der E. meracus-Gruppe durch die nahezu gleichlangen 3. und 4. Glieder der Maxillarpalpen und die äußerst feine und sehr lockere Punktierung des Pronotum gut einzuordnen, so daß nur noch die Abgrenzung zu E. rutilus notwendig ist. Von diesem, außer im völlig verschiedenen Aedoeagus, durch den (bei ausgefärbten Stücken!) basal breit halbkreisförmig geschwärzten Clypeus zu unterscheiden. Bei E. rutilus ist der Clypeus dagegen nahezu ungeschwärzt. 


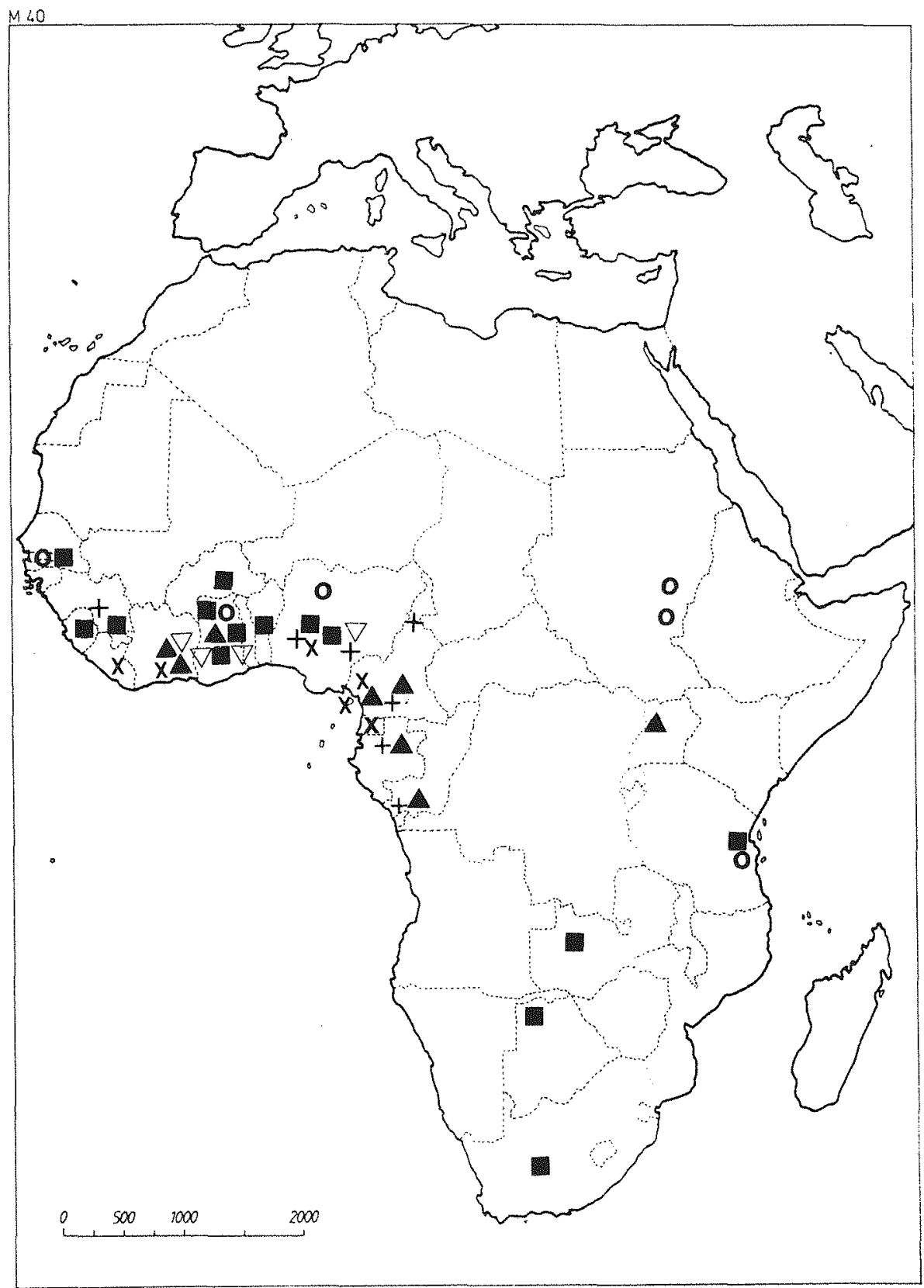

Fig. 15: Geographische Verbreitung von: E. melinus (o), E. meracus (1), E. mergus (4), E. merops $(\mathbf{X})$, E. petersenae $(\nabla)$, E. rutilus $(+)$. 
Beschreibung: Länge: 2,2-2,5 mm.- Ziemlich kurz oval, mäßig gewölbt, lackglänzend schwarz, mit sehr fein punktierter Oberseite, hellem Labrum und hellem Vorderrand des Clypeus sowie gleichlangen 3. und 4. Gliedern der Maxillarpalpen.

Kopf sehr fein, locker punktiert, mit wenigen groben Punkten jeweils auf der Innenseite der Augen. Stirn, Vorderrand der Augen und ein breiter halbkreisförmiger basaler Bereich des Clypeus glänzend schwarz; Labrum und Mundwerkzeuge hell. Das letzte und vorletzte Glied der Maxillarpalpen von gleicher Länge (Fig. 13k). Pronotum äußerst fein, sehr weitläufig punktiert (Punktabstand ca. 5 x Punktdurchmesser), schwarz, lackglänzend, nur der Seitenrand und die Ecken gut begrenzt schmal gelb. Elytren deutlich kräftiger punktiert als das Pronotum; unregelmäßige Reihen gröberer Punkte nicht erkennbar; völlig schwarz, ohne laterale oder apikale Aufhellung. Unterseite und Beine dunkel pechbraun, Tarsen heller. Apikale Ausrandung des Analsternits halbkreisförmig. Aedoeagus charakterisiert durch die häkchenförmig nach außen gekrümmten Paramerenspitzen (ähnlich bei E. merops!) und die auffallend lange Ventralplatte, welche fast das Ende des apikal keulenförmig verbreiterten Medianlobus erreicht. (Fig. 10).

Dertivatio nominis: Lat.: mergus = Taucher.

Verbreitung: W-Afrika, Uganda.

Enochrus (Methydrus) merops BALFOUR-BROWNE, 1958 1958 Enocbrus (Metbydrus) merops J. BALFOUR-BRowNE, p. 54-55.

Locus typicus: W-Afrika (Guinea).

Typenmaterial: Holotypus (männlich): French Guinea; Fouta Djallon, Dalaba, (Mus. Frey, Basel).. PT: 2 o, 4 o (Angola, Elfenbeinküste, Kamerun, Nigeria, Fernando Po; NHM).

\section{Nachweise:}

?Ghana (P): Ashanti region: Kumasi Nhiasu, $6^{\circ} 43 \mathrm{~N} 1^{\circ} 35^{\circ} \mathrm{W}, 330 \mathrm{~m}$, light, 12.6.1967, no. 225, leg. S. Endrödy-Younga (CHG).-?Rep. Kongo (Zaire) ( $(9)$ : Kivu Iranga, 500 m, 18.2.1984, leg. Mühle (CHG). (Aedoeagus Fig. 11; Maxillarpalpen Fig. 13f).

Verbreitung: W-Afrika.

Enochrus (?Enochrus) pellax BALFOUR-BROWNE, 1958 1958 Enocbrus (Enocbrus) pellax BALFOUR-BROWNE, p. 50.

Locus typicus: Guinea (Nimba).

Typenmaterial: Holotypus $\left(0^{*}\right): 3,1 \times 1,65 \mathrm{~mm}$. French Guinea, Nimba; PT: 2 o (MHNP).

Nachweise $10^{*}$ :

Nigeria, Samaru, Dr. S. Endrödy-Younga/ No. 403, light trap, 20.CX.1969 (TMB).

Verbreitung: Westafrika. 
Enochrus (Methydrus) petersenae BALFOUR-BROWNE, 1958 1958 Enochrus (Metbydrus) petersenae J. BALFOUR-BROWNE, p. 51-52.

Locus typicus: W-Africa (Ghana).

Typenmaterial: Holotypus (o): Ghana nt. Agogo, 15 W $6^{\circ} 50^{\prime} \mathrm{N}$; PT: 1 q, ibidem (NHM).

Nachweise:

Ghana: $1 \mathrm{o}^{\star}$ : Ghana, Eastern reg. Boti falls, $30 \mathrm{~km}$ NE Koforidua, leg. Dr. S. Endroedy-Younga/ No. 490, shore washing, 27.XII.1971. (CHG).- Kumasi, 6.7.1967, leg. S., Endrödy-Younga (TMB); 20 Ex.: Ashanti reg. Kumasi, Nhiasu $330 \mathrm{~m}, 6^{\circ} 43^{\prime} \mathrm{N}-1^{\circ} 36^{\prime} \mathrm{W}, 20 . \mathrm{V} .1967$, Nt. 218, at light, leg. Dr. S. Endrödy-Younga (TMB).- Elfenbeinküste: Kafalo/Komoé, 25.4.1988, leg. F.-T.Krell (MNS).- Nigeria: Ile-Ife, 13.7.1988 und 9.- 13.12.1988, leg. F.-T. Krell (MNS, CHG); ibidem 9.7.1988; ibidem 11.7.1988 (MNS).- Sudan: 1 웅 (Equatoria), Nzara, 22.4.1986, leg. Wewalka (NMW).

(Aedoeagus Fig. 3; Maxillarpalpen Fig. 13g).

Verbreitung: Westafrika.

Enochrus (Methydrus) rutilus BALFOUR-BROWNE, 1958 1958 Enocbrus (Metbydrus) rutilus J. BALFOUR-BRoXNe, p. 55.

Locus typicus: W-Afrika (Nigeria).

Typenmaterial: Holotypus ( $\sigma^{*}$ : Nigeria, Ogoia Prov., PT: 4 o, 2 o, ibidem; 1 đ Kamerun, NHM)

\section{Nachweise:}

Gabon: 3 o, 3 \%: Gabon-Makokou à la limiére, 1971, J. Mateu (CHG).- Guinea: 1 q : Seredou, lux, 4.4.1975, leg. A. Zott (CHG)-- Kamerun: 1 Ex.: N.-Kamerun, Victoria, Strunk S. (CHG).- 1 q: Cameroon: $65 \mathrm{~km}$ NE Kribi, Bidjouka, Secondary Forest, at stream, at light, 4.I.1978, Loc. No. 13/ Lund Univ. Syst. Dept., Sweden Cametoon Exp. 1977-78. (ZML).- Kongo: $1 \delta^{*}, 1$ \%: Soil-Zool. Exp. Congo-Brazzaville, Bouenza catarract/30.1.1963, No. 308, sifted in float, leg. Endroedy-Younga. (TMB, CHG).- Nigeria: Ogoja County Rev. Meyer A 9/86, stagnant pool, 5.4.1962; 4 \%: Ile-Ife, 9.VII.1988, leg. F.-T. Krell (MNS, CHG). (Aedoeagus Fig. 6; Maxillarpalpen Fig. 13 I).

Verbreitung: Westafrika.

\section{Literatur}

Batfour-Browne, J. 1952: Mission A. Villiers au Togo et au Dahomey (1950). VII. Coléoptères Hydrophilides. - Bulletin d'Institut francoise d'Afrika noire 14: 126-139.

Balfour-Browne, J. 1958: New West Africat Enochrus (Coleoptera: Hydrophilidae), - Proceedings of the Royal entomological Society of London (B) 27 (3-4): 49-55.

Balfour-Browne, J. 1959: Dr. Jan Bechyné expedition to French Guinea, 1951 (Hydrophilidae). - Entomologische Arbeiten des Museums Frey 10 (2): 1: 302-320.

GrEEN, J. W. (ined.): Key to the Nearctic Species of the Genus Enochrus (Coleoptera: Hydrophilidae), p. 58-67.

GUNDERSEN, R. W. 1977: New species and taxonomic changes in the genus Enocbrus (Coleoptera: Hydrophilidae). - The Coleopterists Bulletin 31 (3): 251-272.

HANSEN, M. 1999: Hydrophiloidea, in: World Catalogue of Insects, Vol. 2: 416 pp. Apollo Books.

Hebauer, F. 1988: Hydrophiloidea aus Namibia. - Bonn. zool. Beitr. 39 (2/3): 153-161.

HEBAUER, F. 1995: Neues zu den Acidocerina HANSEN (Helocharae D'ORCHYMONT) der indomalaiischen Region (Coleoptera, Hydrophilidae). - Acta coleopterologica 11 (3): 3-14, München. 
HEBAuER, F. 1998: Taxonomische Studien zur Hydrophiliden-Gattung Enochrus THOMSON, 1859. - 1.Teil : Zwei afrikanische Außenseiter aus den Untergattungen Enochrus s. str. und Hydatotrephis MAC LEAY. (Coleoptera: Hydrophilidae). - Ent. Blätter 94: 143-146, Jena.

HORN, G. H. 1890: Notes on some Hydrobiini of Boreal America. - Transactions of the American Entomological Society, 17: 237-278.

Milter, D. C. 1964: Notes on Enochrus and Cymbiodyta from the Pacific Northwest (Coleoptera, Hydrophilidae). - Coleopterists Bulletin 18: 69-78.

OrChYMont, A. d'1939: Contribution à l'ètude des Palpicornia XIII. - Bull. Annls. Soc. Ent. Belg., 79; 357-378.

WATTS, C. H. 1998: Revision of Australian Enochrus THOMsON (Coleoptera: Hydrophilidac). - Records of the South Australian Museum 30 (2): 137-156.

YounG, F. N. 1954: The Water Beetles of Florida. - University of Florida press, Gainesville, p. 174-183.

\section{Anschrift des Verfassers:}

PD Dr. Franz Hebauer

Ulrichsberg 7

D-94539 Grafling

Deutschland

\section{Besprechungen}

SChÄffLER, O: Tagfalter. Teil 12: Papilionidae VI, Otnithoptera, Text, Tafeln. - Keltern: Goecke \& Evers, 2001. - 20 S., 52 Farbtaf. - (Schmetterlinge der Erde / BAUER, E. \& FrAnKKanBACH, T. [Hrsg.]. ISBN 3-931374-82-3

Der vorliegende Teil in dieser Reihe ist als Supplement zu der 1997 erschienenen Bearbeitung der Gattung durch B. VON KNÖTGEN konzipiert. Es werden neben den dort fehlenden Unterarten und Formen einige neue Taxa (1 Unterart, 3 Formen) beschrieben. Informativ sind die im Textteil vorgelegten Kartenausschnitte, die eine Lokalisierung der typischen Fundorte in der Inselwelt um Neuguinea erleichtern.

Auf den, wie schon in den vorhergehenden Teilen diese Serie hervorragenden, Farbtafeln sind die behandelten Taxa in beiden Geschlechtern, oft auch mit Ober- und Unterseite dargestellt.

Jeder, der sich mit den Rhopaloceren befaßt, sei es als Sammler, sei es als Systematiker, wird mit Interesse auf die weiteren Teile dieser Reihe warten.

R. GAEDIKE

LAŠTƯVKA, Z. \& LAŠTƯVKA, A.: The Sesiidae of Europe. - Stenstrup: Apollo Books, 2001 - 245 p.: 107 plates, 9 colour plates. - ISBN 87-88757-52-8

In den letzten Jahten sind über diese Lepidopterenfamilie mehrere zusammenfassende Darstellungen etschienen, so dass man geneigt ist, sich über eine erneute Bearbeitung etwas zu wundern. Gerade aber das Vorhandensein von Büchern, die die Determination dieser biologisch so interessanten Gruppe ermöglicht haben, führte nicht nur zu einer starken Zunahme von neuen Kenntnissen zur Verbreitung und Biologie bekannter, sondern auch zur Entdeckung zahlreicher neuer Taxa. Mit ein wesentlicher Grund dafür ist auch die Weiterentwicklung der Methode des Fangs mit Pheromonen.

In vorliegendem Buch werden die gegenwärtig vorhandenen Informationen über die 107 europäischen Sesiidae zusammengefaßt. 